\title{
AVALIAÇÃO DE DANOS DE ADULTOS DE COSTALIMAITA FERRUGINEA (FABRICIUS) (COL.: CRYSOMELIDAE) EM EUCALYPTUS SPP. NA REGIÃO DE PRESIDENTE PRUDENTE, SP
}

\author{
S.M.N.M. Montes ${ }^{1}$, M.E. Sato ${ }^{2}$, A. Raga ${ }^{2}$, L.C. Cerávolo ${ }^{1}$
}

${ }^{1}$ Polo Regional da Alta Sorocabana, Rod. Raposo Tavares, km 561, CEP 19015-970, Presidente Prudente, SP, Brasol. E-mail: soniamontes@apta.sp.gov.br

\section{RESUMO}

\begin{abstract}
Os danos provocados pelo besouro-amarelo Costalimaita ferruginea em folhas de Eucalyptus camaldulensis, E. citriodora, E. pellita, E. urophylla e E. urograndis foram avaliados em floresta instalada no Município de Presidente Prudente, Estado de São Paulo. Para avaliação dos danos de C. ferruginea foram coletadas 14 folhas adultas por planta, em duas plantas por parcela. As folhas foram escolhidas ao acaso, na altura de 1,50 m do solo. As parcelas eram constituídas de aproximadamente 120 plantas, com cinco repetições. $\mathrm{O}$ dano de C. ferruginea foi estimado por meio de notas visuais em todas as folhas coletadas, baseadas em uma escala diagramática de danos que variou de 0 a $100 \%$ (Notas 1 a 8 ). As médias das notas dos danos causados pelo besouro-amarelo em folhas de eucalipto variou entre 1,36 e 6,04. As folhas de E. camaldulensis foram as menos danificadas pelos adultos de $C$. ferruginea, enquanto que as de E. urophylla e E. urograndis foram as mais preferidas para alimentação, com danos semelhantes entre si. Os danos em folhas de E. citriodora e E. pellita foram intermediários, sendo esta espécie significativamente menos atacada que a primeira.
\end{abstract}

PALAVRAS-CHAVE: Insecta, praga, eucalipto.

\section{ABSTRACT}

EVALUATION OF DAMAGES CAUSED BY COSTALIMAITA FERRUGINEA (FABRICIUS) (COL.: CRYSOMELIDAE) ON EUCALYPTUS SPP. IN PRESIDENTE PRUDENTE REGION, SÃO PAULO STATE, BRAZIL. The damage caused by feeding of eucalyptus yellow beetle Costalimaita ferruginea on leaves of Eucalyptus camaldulensis, E. citriodora, E. pellita, E. urophylla and E. urograndis was evaluated in a forest installed in the municipality of Presidente Prudente, state of São Paulo, Brazil. The percentage of defoliation was evaluated by collection of fourteen mature leaves per tree from two trees per plot. The leaves were randomly chosen up to $1.50 \mathrm{~m}$ high. The plots consisted of approximately 120 trees with five replications. The visible foliar damage was estimated using a score scale (1 to 8 ) ranging from 0 to $100 \%$ of defoliation. Considering all Eucalyptus species, the score average for the defoliation due to eucalyptus yellow beetle infestation ranged from 1.36 to 6.04. The leaves of E. camaldulensis were the least infested C. ferruginea adults. E. urophylla and E. urograndis showed the highest preference for $C$. ferruginea feeding, with similar scores for damages. The leaf damages in E. citriodora and E. pellita were considered intermediate, with lower score for E. pellita.

KEY WORDS: Insecta, pest, eucalyptus.

O reflorestamento é um dos setores com maior crescimento no Brasil, principalmente através da exploração de espécies de Eucalyptus, que no Estado de São Paulo abrange 312.939 hectares, correspondendo a $27,3 \%$ da área total cultivada com eucalipto em nosso país (Estatísticas, 2005). A exploração comercial com espécies de eucalipto está espalhada em todas as regiões paulistas, algumas delas de forma mais extensiva, destinadas às indústrias de papel e celulose, fato que tem provocado frequentes problemas com insetos pragas. Segundo GraHAM (1956), a injúria causada por insetos pragas em árvores pode provocar redução no crescimento, degradação da madeira ou deterioração das toras deárvores mortas.

Os besouros desfolhadores (Coleoptera) constituem o terceiro grupo de insetos pragas mais relevante para a silvicultura brasileira, e sua importância vem crescendo em função do crescimento de novos registros, principalmente na cultura de eucalipto.

O crisomelídeo Costalimaita ferruginea (Fabricius) é a principal espécie de besouro desfolhador de importância econômica para o setor florestal brasileiro

${ }^{2}$ Instituto Biológico, Centro Experimental Central, Campinas, SP, Brasil. 
e o quarto inseto praga mais importante para a produção de eucalipto (SILVA, 1992; ANJOS, 2005), sendo conhecido como besouro-amarelo. Os adultos de C. ferruginea têm hábito noturno, alimentam-se das folhas, deixando-as perfuradas ou rendilhadas (JUNQUEIRA, 1962), podendo causar um pesado desfolhamento em casos de ataque intenso, principalmente em plantas de eucalipto em desenvolvimento. Também podem roer ponteiros e frutificações dos eucaliptos (SILVA, 1992). Os adultos apresentam de 5 a $6,5 \mathrm{~mm}$ de comprimento e de 3 a 3,5 mm de largura. A postura, em média de 91 ovos, é feita no solo, onde as larvas do besouro-amarelo completam o seu ciclo, alimentando-se de raízes. As maiores infestações tem sido observadas de novembro a março (MARICONI, 1956; Anjos et al., 1986; SILVA, 1992).

C. ferruginea ocorre desde a Amazônia até a Argentina (LimA, 1955), em quase todos os estados brasileiros (SILVA, 1992). No Estado de São Paulo já havia sido referido nas décadas de 1950 e 1960 em quinze municípios (MARICONI, 1956; JUNQUEIRA, 1962), alguns deles na região oeste. Além de espécies de eucalipto, o besouro-amarelo ataca abacateiro, algodoeiro, araçazeiro, cajueiro, camu-camu, goiabeira, jambeiro, jambolão, jabuticabeira, manacá, macieira, mangueira, pitangueira, araçá-do-mato (Psidium guineense) e videira (LIMA, 1955; MARICONI, 1956, 1985; JunQUEIRA, 1962; Silva et al., 1968; SILVA, 1992; GALlO et al., 2002; MAUÉs; COUTURIER, 2002).

Praga-chave de mudas de eucalipto, C. ferruginea também causa redução na produtividade e custos consideráveis para o seu controle em plantas de eucalipto em desenvolvimento. O objetivo da presente pesquisa foi medir os danos foliares causados pelo besouro amarelo em diferentes espécies de eucalipto, em condições de campo, na região de Presidente Prudente, região oeste do Estado de São Paulo.

O experimento foi conduzido na fazenda sede do Polo Regional de Desenvolvimento Tecnológico dos Agronegócios da Alta Sorocabana, APTA, localizada no Município de Presidente Prudente, Estado deSão

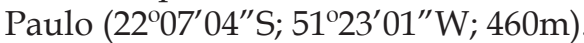

A área onde o estudo foi conduzido, constituída de quatro espécies e um híbrido, tinha um total de 650 plantas de Eucalyptus citriodora Hook, 600 plantas do híbrido E. urograndis, 650 plantas de E. urophylla S.T. Blake, 1000 plantas de E. camaldulensis Dehnh. e 300 plantas de E. pellita F. Muel., totalizando 3200 plantas, com espaçamento de 3,0 X 2,0 m. As plantas de $E$. citriodora, E. urophylla e E. urograndis apresentavam 12 meses de idade e as de E. camaldulensis e E. pellita tinham 2 meses e meio no campo. $\mathrm{O}$ ataque inicial do besouro-amarelo nos talhões de eucalipto foi observado em 10/10/2005, com intensidade crescente.

Para avaliação dos danos de C. ferruginea, no dia 19/11/2005 foram coletadas 14 folhas adultas por planta, em duas plantas por parcela. As folhas foram escolhidas ao acaso, na altura de 1,50 $\mathrm{m}$ do solo, coletando aquelas localizadas no terceiro par de folhas da extremidade para o interior da planta e abrangendo os quatro quadrantes. As parcelas eram constituídas aproximadamente de 120 plantas. $\mathrm{O}$ delineamento utilizado foi inteiramente casualizado, com cinco repetições e cinco tratamentos (espécies / híbrido de eucalipto).

O dano de C. ferruginea foi estimado por meio de notas visuais em todas as folhas coletadas, baseadas em uma escala diagramática que variou de 0 a $100 \%$ (Fig. 1), onde:

Nota $1(\mathrm{~N} 1)$ = folhas sem nenhum dano $(0 \%)$;

Nota 2 (N 2) = folhas com 1 a $5 \%$ de dano;

Nota $3($ N 3) = folhas com 6 a $10 \%$ de dano;

Nota 4 (N 4) = folhas com 11 a $20 \%$ de dano;

Nota 5 (N 5) = folhas com 21 a $40 \%$ de dano;

Nota 6 (N 6) = folhas com 41 a $60 \%$ de dano;

Nota 7 (N 7) = folhas com 61 a $80 \%$ de dano;

Nota 8 (N 8) = folhas com 81 a $100 \%$ de dano.

As médias das notas foram analisadas pelo programa estatístico "Statistical Package for the Social Sciences (SPSS 2000 - SPSS para Windows, versão 10.0.)", através do teste $\mathrm{F}$ e comparadas pelo teste de Tukey a $5 \%$ de probabilidade.

As médias das notas dos danos, causados pelo besouro-amarelo em folhas de eucalipto, oscilaram entre 1,36 e 6,04. Segundo o teste de comparação das médias das notas, as folhas de E. camaldulensis foram as menos danificadas pelos adultos de C. ferruginea, enquanto que as de E. urophylla e E. urograndis foram as mais preferidas para alimentação, com danos semelhantes entre si. Os danos em folhas de E. citriodora e E. pellita foram intermediários, sendo que esta espécie foi significativamente menos atacada que a primeira (Fig. 2). Segundo Silva (1992), adultos do besouro-amarelo foram observados atacando folhas de 24 espécies e de dois híbridos de Eucalyptus no Brasil, sendo os danos mais comuns em E. grandis e E. urophylla. Segundo aquele autor, E. camaldulensis, E. microcorys F. Muell e E. tereticornis Smith são pouco atacadas por C. ferruginea.

Embora não avaliada neste experimento, provavelmente a diferença na preferência entre espéciese híbridos de eucalipto esteja relacionada com as características físico-químicas das folhas, onde algumas delas propiciam propriedades fagoestimulantes aos adultos do besouro-amarelo, principalmente folhas mais tenras e altos teores de nitrogênio (OHMART et al., 1985). Além desses fatores, existem diferenças na constituição de compostos secundários das folhas das espécies de eucalipto, como taninos, outros compostos fenólicos, gorduras eóleos essenciais, que influenciam negativamente o processo de herbivoria (OHMART; EdWARds, 1991). BAKER et al. (2002) concluíram que o crisomelídeo Chrysophthartabimaculata (Olivier), uma praga de eucaliptos na Austrália, tem 
melhor performance quando se alimenta de folhas de Eucalyptus nitens Maiden quando comparado com folhas E. regnans F. Muell. Pelo fato daquela espécie de eucalipto apresentar folhas com maior valor nutricional e menores níveis de alguns óleos, que provocam altos níveis de desfolhamento.

Na comparação entre as espécies de eucalipto, E. camaldulensis apresentou a maior porcentagem de folhas sem danos $(76,4 \%)$, diferindo dos demais materiais (Tabela 1). Essa espécie não apresentou folhas danificadas por C. ferruginea com notas acima de 5 . Com exceção das notas 1 , 2 e 4 , as notas atribuídas aos danos em folhas de E. camaldulensis foram semelhantes as de E. pellita. As porcentagens de folhas de E. camaldulensis com notas 1 e 2 foram significativamente diferentes entre si e superiores às demais notas atribuídas aos danos nessa espécie.

Todas as folhas amostradas de E. urophylla e do hibrido E. urograndis apresentaramalgum danoe para essehíbrido, todas as folhas foram avaliadas com pelo menos nota 3. Aproximadamente, $76 \%$ das folhas de E. urophylla apresentaram notas iguais ou acima de 5 . As porcentagens de folhas com notas 5, 6 e 7, dentro de E. urophylla e de E. urograndis, foram semelhantes entre si, alcançando $75 \%$ e $65 \%$ do total de folhas coletadas para esses materiais, respectivamente. Segundo levantamento conduzido por Silva (1992) em sete estados brasileiros, $27,9 \%$ dos ataques de $C$. ferruginea foi registrado em E. grandis e $23,8 \%$ em E. urophylla. Isso demonstra a preferência do besouro-amarelo por essas duas espécies e seus híbridos.

Tabela 1. Porcentagem de folhas para cada nota de dano de C. ferruginea em diferentes espécies/híbrido de eucalipto. Presidente Prudente, SP, nov/2005.

\begin{tabular}{|c|c|c|c|c|c|c|c|c|c|}
\hline \multirow{2}{*}{ Espécie/híbrido } & \multicolumn{8}{|c|}{ Nota $^{1}$} & \multirow{2}{*}{$\begin{array}{l}\text { C.V. } \\
(\%)\end{array}$} \\
\hline & 1 & 2 & 3 & 4 & 5 & 6 & 7 & 8 & \\
\hline E. camaldulensis & 76,4 с E & 15,0 c D & $5,0 \mathrm{abC}$ & 2,9 a BC & 0,7 a $\mathrm{AB}$ & 0,0 a $\mathrm{A}$ & 0,0 a $\mathrm{A}$ & 0,0 a $\mathrm{A}$ & 27,5 \\
\hline E. citriodora & 2,1 a $A$ & 8,6 b B & 18,6 с C & 15,0 b BC & $20,0 \mathrm{~b} \mathrm{C}$ & $15,0 \mathrm{~b} \mathrm{BC}$ & $13,6 \mathrm{~b} \mathrm{BC}$ & $7,1 \mathrm{ab} \mathrm{A}$ & 17,9 \\
\hline E. pellita & $43,6 \mathrm{~b} \mathrm{~F}$ & $24,3 \mathrm{~d} E$ & $11,4 \mathrm{bCD}$ & $12,9 \mathrm{~b} \mathrm{D}$ & 5,0 a BC & 2,1 a $A B$ & 0,7 a $\mathrm{A}$ & 0,0 a $\mathrm{A}$ & 21,2 \\
\hline E. urophila & 0,0 a $\mathrm{A}$ & 0,0 a $A$ & 2,1 a $\mathrm{AB}$ & $9,3 \mathrm{ab} B$ & 21,4 b C & $30,7 \mathrm{~d} C$ & 22,9 c C & $13,6 \mathrm{~b} \mathrm{AB}$ & 37,4 \\
\hline E. urograndis & 0,0 a $\mathrm{A}$ & $5,0 \mathrm{~b}$ BC & 9,3 b CD & $10,0 \mathrm{ab} C D$ & $17,9 \mathrm{~b} \mathrm{DE}$ & 21,4 c E & 25,7 c E & $10,7 \mathrm{ab} B$ & 23,9 \\
\hline C.V. $(\%)$ & 16,5 & 27,1 & 32,4 & 30,3 & 21,3 & 15,4 & 21,3 & 29,5 & \\
\hline
\end{tabular}

Médias seguidas de letras minúsculas diferentes na coluna e de letras maiúsculas na linha diferem entre si, pelo teste de Tukey a

$5 \%$ de probabilidade.

${ }^{1}$ Dados Originais. Dados transformados em arc seno x/100, para fins de análise estatística.
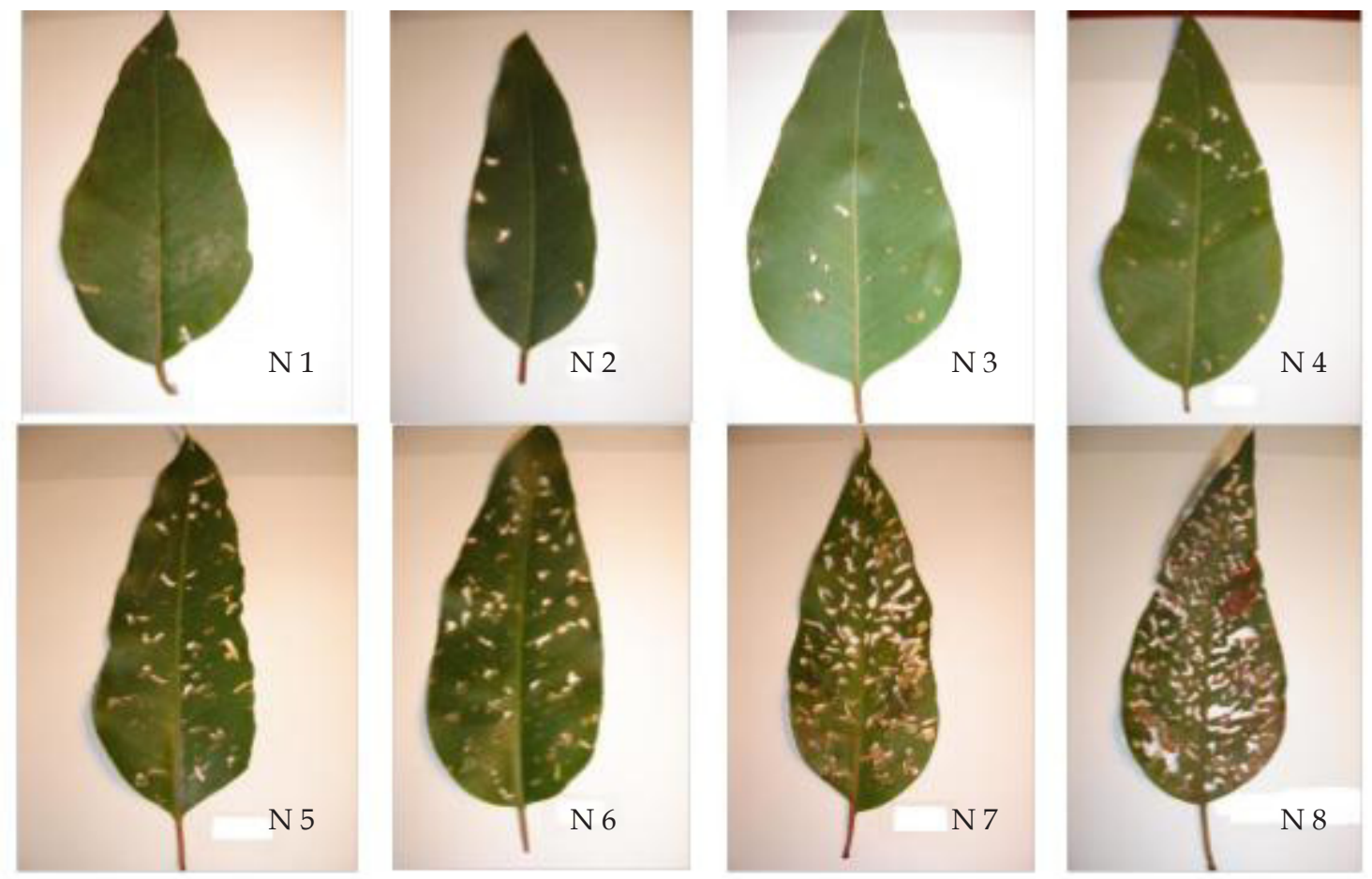

Fig. 1 - Escala diagramática de danos (Notas 1 a 8) causados por C. ferruginea em folhas de eucalipto. 


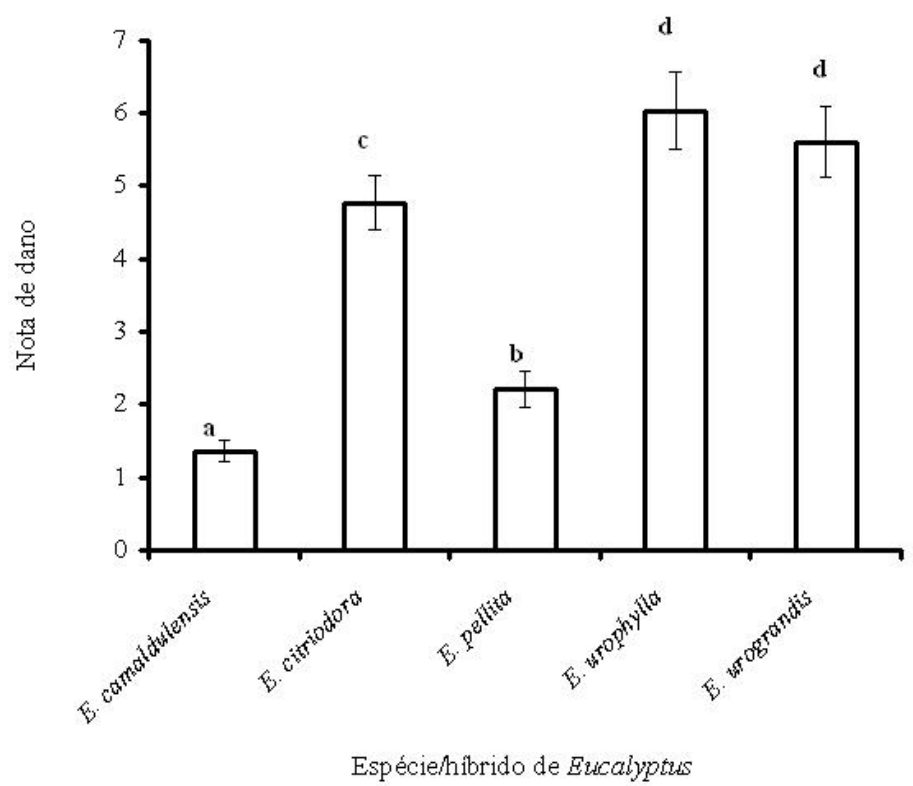

Fig. 2 - Nota de dano de C. ferruginea em diferentes espécies/híbrido de eucalipto. Média de dano \pm DP (desvio padrão). Colunas com letras diferentes diferem entre si, pelo teste de Tukey a $5 \%$ de probabilidade.

Com exceção das notas 2, 3 e 6, os danos apresentados pelo besouro-amarelo em E. urophylla e E. urograndis foram semelhantes entre si. E. pellita apresentou $43,6 \%$ das folhas sem danos, $24,3 \%$ da folhas com nota 2 e nenhuma folha com nota 8.

E. citriodora mostrou-se com maior distribuição de frequências, com folhas em todas as notas. As porcentagens de folhas dessa espécie com notas de dano de $C$. ferruginea entre 3 e 7 foram semelhantes entre si, que somadas alcançaram $82,2 \%$.

Pode-se concluir que dentre os materiais de eucalipto avaliados, os adultos de C. ferruginea têm preferência para se alimentar em folhas de $E$. urophylla e do híbrido E. urograndis, sendo as folhas de E. camaldulensis pouco atacadas pelo besouroamarelo.

\section{REFERÊNCIAS}

ANJOS, N. dos Besouros desfolhadores de plantações florestais no Brasil. Universidade Federal de Viçosa, MG. Disponível em: $<$ http:/ /www.insecta.ufv.br $>$. Acesso em: 24 nov. 2005.

ANJOS, N.; SANTOS, G.P.; ZANÚNCIO, J.C. Pragas do eucalipto e seu controle. Informe Agropecuário, v.12, n.141, p.50-58, 1986.

BAKER, S.C.; ELEK, J.A.; CANDY, S.G. Comparison of feeding efficiency, development time and survival of Tasmanian eucalyptus leaf beetle larvae Chrysophtharta bimaculata (Olivier) (Coleoptera: Chrysomelidae) on two hosts. Australian Journal of Entomology, v.41, p.174-181, 2002.
ESTATÍSTICAS - Situação florestal: Área (em ha), com eucalipto, pinus, seringueira e vegetação natural nas Unidades de Produção Agrícola, por bacia hidrográfica e município do Estado de São Paulo, 1996/2004. Florestar Estatístico, v.8, n.17, p.41-61, 2005. Disponível em: $<$ http:www.fflorestal.sp.gov.br>. Acesso em: 5 fev. 2006.

GALLO, D.; NAKANO, O.; SILVEIRA NETO, S. CARVALHO, R.P.L.; BAPTISTA, G.C.; BERTI FILHO, E.; PARRA, J.R.P.; ZUCCHI, R.A.; ALVES, S.B.; VENDRAMIM, J.D.; MARCHINI, L.C.; LOPES, J.R.S.; OMOTO, C. Manual de entomologia agrícola. Piracicaba: Fealq. 2002. 920p.

GRAHAM, S.A. Ecology of forest insects. Annual Review of Entomology, v.1, p.261-280, 1956.

JUNQUEIRA, G.M. Nota sobre o "besouro amarelo dos eucaliptais". O Biológico, São Paulo, v.28, p.326-328, 1962.

LIMA, A.C. Insetos do Brasil - Coleópteros. Rio de Janeiro: Escola Nacional de Agronomia, 1955. Tomo 9, Capítulo 29, 289p.

MARICONI, F.A.M. Alguns besouros depredadores de eucaliptos na região de Piracicaba. O Biológico, São Paulo, v.22, p.1-14, 1956.

MARICONI, F.A.M. Inseticidas e seu emprego no combate às pragas. São Paulo: Livraria Nobel, 1985. 466p.

MAUÉS, M.M.; COUTURIER, G. Biologia floral e fenologia reprodutiva do camu-camu (Myrciaria dúbia (H.B.K.) McVaugh, Myrtaceae) no estado do Pará, Brasil. Revista Brasileira de Botânica, v.25, p.441-448, 2002. 
OHMART, C.P.; STEWART, L.G.; THOMAS, J.R. Effects of food quality, particularly nitrogen concentrations, of Eucalyptus blakelyi on the growth of Paropsis atomaria (Coleoptera: Chrysomelidae). Oecologia, v.65, p.543-549, 1985.

OHMART, C.P.; EDWARDS, P.B. Insect herbivory on Eucalyptus. Annual Review of Entomology, v.36, p.637-657, 1991.

SILVA, A.G.A.; GONÇALVES, C.R.; GALVÃO, D.M.; GONÇALVES, A.J.L.; GOMES, J.; SILVA, M.N.; SIMO-

NI, L. Quarto catálogo dos insetos que vivem nas plantas no
Brasil - seus parasitos e predadores. Rio e Janeiro: Ministério da Agricultura, 1968. 622p.

SILVA, N.A. Taxonomia, ciclo de vida e dinâmica populacional de Costalimaita ferruginea (Fabr., 1801) (Coleoptera: Chrysomelidae) praga de Eucalyptus spp. 1992. 165p. Tese (Doutorado - Entomologia) - Escola Superior de Agricultura "Luiz de Queiroz" Universidade de São Paulo, Piracicaba, 1992.

Recebido em 3/9/10

Aceito em 20/6/12 